\title{
A New Algorithm for Shape Detection
}

\author{
Hewa Majeed Zangana \\ Department of Computer Science / College of Computer Science and IT / Nawroz University / Kurdistan Region \\ of Iraq
}

\begin{abstract}
Humans usually have some default shapes which the eye could recognize and know what they are, so vision is an important thing in human knowledge. The same operation applied in machines and computers because the software must recognize the shape before doing any operation on it. In the current paper we proposed a new algorithm for detecting shapes and even detecting the kind of shapes. In addition, the proposed system detected all the types of shapes which are known usually, and the system depends on the concept of dividing the image into areas where some areas will contain the shape and others will not contain the shape and after that the system will detect the factor of shape in order to recognize the kind of shape. The proposed algorithm has been applied on many photos which contain many types of shapes and the results were great and it could detect all of the shapes given in photos.
\end{abstract}

Keywords: recognition shapes.

\section{Introduction}

In the science of image processing and the science of object detection the operation is always trying to detect an object and it requires to knew the all kind of shapes and objects in order to detect them and there are some software are looking for only triangle or only the circle in the whole photo given as an input to the system. In the eye of humans also need to detect the shape which is in front them from the database which stored in the brain of humans and they need to recognize the shape before doing any other operation or step. Researchers of detecting objects are agreeing that the information of area in a photo are important to extract the thing which called perceptual unit. Here some features could be detected and also some texture as properties of signal might be sent in order to assist the system when taking a decision to any point detected if does it related to any shape or does not related to it. In the photos which have been taken randomly contain some shapes like rectangle could play a very important role as information to the shape itself which maybe a vehicle in this case. Other features of shapes just like the shape of human eye could be useful also because this kind of feature could help in detecting the human face because it has the same shape in all faces where other shapes could be related to any other object. In computer science, many researchers are focusing on shape detection because the shape is playing important role to recognize an object. Even in medical science, detecting shape is an important step to specify the diseases especially in analyzing image for example the shape of organ maybe useful in specifying the diseases. Usually we are looking for known and normal shapes but sometimes if the shape is incomplete or the shape is looks like another shape, here we could not recognize which shape is it, so the information of the shape are important due to our vision system needs the complete information of the shape in order to recognize it. Specifying the shape is not that big problem but also it is not so easy to recognize and that is why we need the complete information of the shape. In computer science nowadays, retrieving photos became one of the most things of the interesting of computer vision especially because of the huge number of graphical data which have been stored last years. In these types of information and data the process of analysis depends on the operation of recognizing the graphics in order to let the photo be classified especially when the symbols are available. Generally, there are many types of methods of detection and recognition depending on the kind of information. The approaches of statistic can be used for analyzing the photos and they are really accurate but took long time where if we try the structural approaches we will gain more time because it will be faster than the statistical ones but here we must apply them very carefully to make them accurate. In object recognition, detecting the shape of object and recognize it is very important because it will help the system to recognize the object itself in the given photo.

\section{Literature Review}

One of the researchers supposed an approach for detecting and recognizing shapes like triangle and square and circle in the photo by using an algorithm, and that algorithm was working by utilizing the value of intensity from the photo which has been input and applied then the thresholding in order to get the binary image. A filter step applied after that to erase the noise and after that an operation is used to locate the edges of shape and another approach is used to erase the edges which are not needed because these edges could be related to something else and for decrease the false recognition. The density of the region helped the decision of shapes. 
The results of this algorithm represent an accuracy of $85 \%$ by using this approach and when it applied on a data base has been selected before [8].

Another researchers proposed another method for detecting shapes from the photo and this method was depending on the surface of many objects by touch and in this method some objects used to detect and recognize the shapes like an object has double edges and a planar surface and an object with only single edge and cylindrical and some more. In addition, thresholding has been applied in multilevel for denote the edge's surface in high area's pressure and this high pressure gained uniquely for many classes of object and another area's descriptors applied in order to describe the area's high pressure uniquely. For the purpose of classification, the area's descriptors applied as needed features. For classifying the shape of object, the linear support vector machine has been applied. The accuracy of classifier gave $92 \%$ when applied in the environment could has a noise. For proving the efficiency of the operation of classification, statistical tests applied [7].

Other researchers proposed a methodology depended on the studying and analyzing the shape especially the shape of curves which are continuous and closed in the shape by applying the geodesic paths where the curve angle has been represented. They studied the tree shape where crowns in the colored photos. They extract the crowns of the tree for make the classification level. They found in this study the problem of multiple objects when applying the extraction step on them especially when the photo has a very high resolution [3].

Other researchers supposed another approach for detecting the class of object in the photos depending on the global shape, where they measure the distance of the elastic shape where it matches and derived which is difficult to rotate and scale. For finding the boundaries they begin with segmenting the photo and they explored the potential space of object boundaries because they have the high similarity in template of the class of shape of the object in order to be recognized. The proposed approaches gave accuracy equal to $83 \%$ in detecting the shapes from photos in the selected database [6].

\section{Proposed Algorithm}

We have proposed a new algorithm for recognizing and detecting shapes in the photos. The proposed algorithm has been developed to recognize and detect many different shapes given in any colored photo and even in black white photo and the approach of the proposed algorithm is depending on the gray scale photos and even the colored photos given as input for the proposed system will be convert to gray scale photo and for enhancing the photo, a contrast limited adaptive histogram equalization applied on small areas in the photo and it has been enhanced by the histogram equalization, so our first level is to enhance the given photo and this technique of histogram equalization is known already for analyzing the photo and enhance it. So we have applied this technique on the photo which is in gray scale level and after that we have applied the gaussian process of filtering which used and applied on the photo which resulted from the step of enhancing the photo in order to decrease the noising and this process will be applied as following:

$$
G(x, y)=\frac{1}{2 \pi \sigma^{2}} \exp \left(\frac{x^{2}+y^{2}}{\sigma^{2}}\right)
$$

After that we have adjust the photo in the value of intensity and the map of color in order to increase the contrast of the resulted photo from equation (1) and this was the first step as we said for enhancing the photo and after that we are going to exchange the pixels of photo which are smaller and equal the threshold value to value 1 which is the white color and the other pixels of photo will be changed to value 0 which is the black color and this step will give us the binary photo and in this binary photo we can notice that the black color will represent the background of the photo and the white color in the photo will represent the shape or the object and this step will be shown in Figure 1 which represent the photo at these levels.

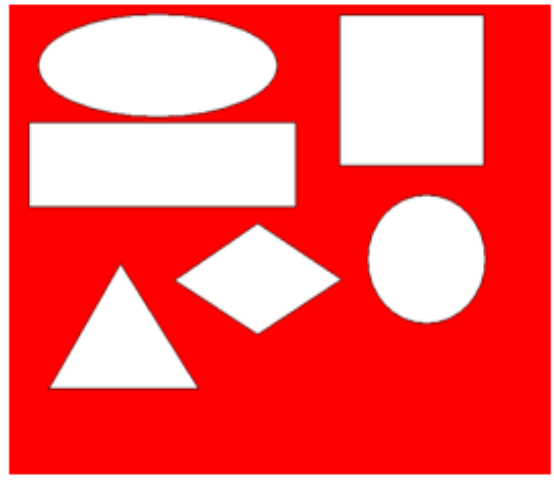

(A)

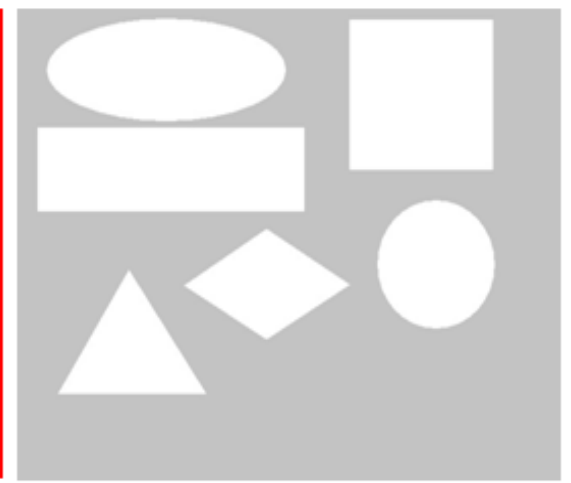

(B) 


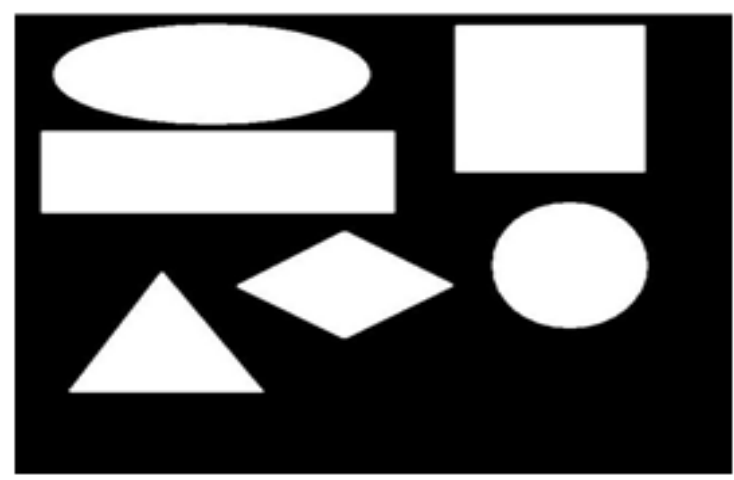

(C)

Fig. 1: A: Represent the input colored photo. B: Represent the gray scale level.

$\mathrm{C}$ : Represent the binary photo

In the proposed algorithm, we are tracking the boundaries of every shape given in the photo and after that we are going to label all the areas detected in the binary photo depending on the algorithm of labeling photo as represented in Figure 2 and it is going to scan the whole pixels of the photo and after that it is going to assigning primary labels to the pixels have value not equal to zero and recording the label equivalences in a union find table and using the algorithm of union find to record the equivalence classes where the algorithm of union find is applied for maintaining a set of non overlapping number from a limited area of elements. After that we are going to label again the pixels depending on the equivalence classes which have been resolved.

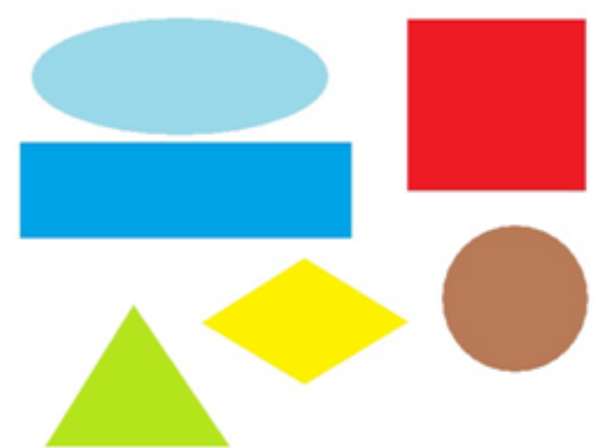

Fig. 2: Photo Labeling

After that we are going to detect and recognize every area which has been labeled from the other similar area by using the accounting of parameter of the shape factor where the shape factor is applied to detect and recognize the area which has been labeled even if it is a known shape or not and after that it will represent the kind of the detected shape in the case of known shape and this can be done by applying the following equation:

$$
\text { shape factor }(\mathrm{SF})=\frac{\text { area }}{(\text { diameter })^{2}}
$$

Here the diameter represents the highest distance among two pixels in any labeled area or we can say in the exactly boundary of area which has been labeled and the area represent the labeled area or region and the shape factor's value represents a specific kind of a shape depending on Table 1 and Figure 3 represents the working of the algorithm we proposed in this paper which is detecting the shapes given in the input photo. 


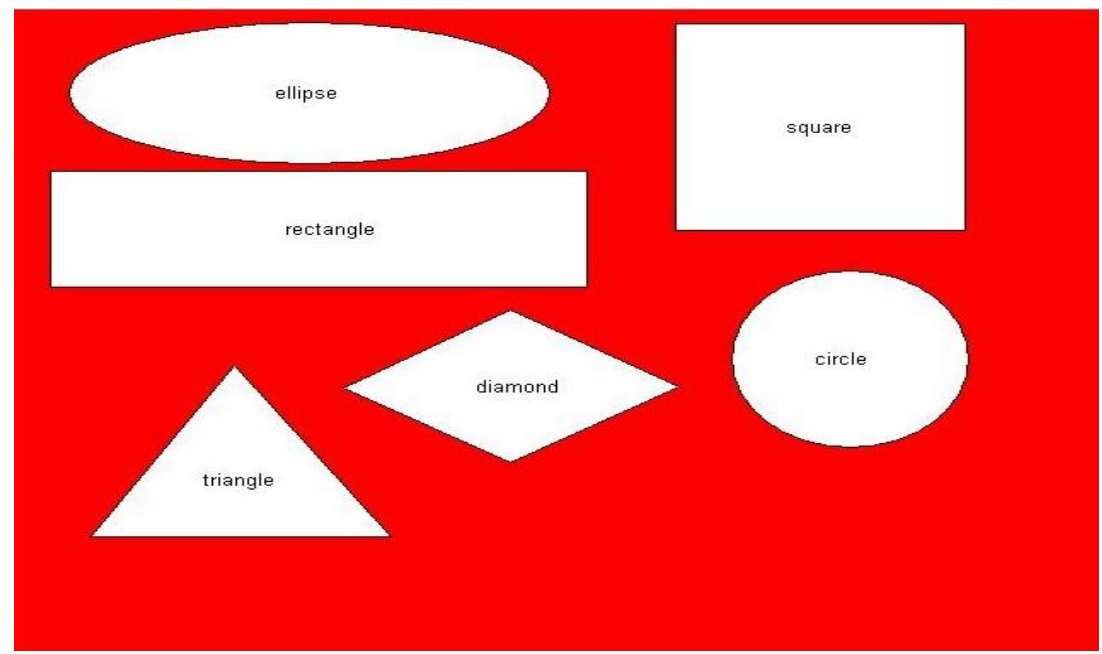

Fig. 3: Detected and Recognized Shapes

The following table represents some values of the shape factor:

\begin{tabular}{|l|lll|}
\hline Shape Type & \multicolumn{3}{|l|}{ Shape Factor (SF) } \\
\hline Circle & $0.7 \leq$ & SF & $\leq 0.8$ \\
\hline Square & $0.484 \leq$ & SF & $\leq 0.55$ \\
\hline Rectangle & $0.2 \leq$ & SF & $\leq 0.3$ \\
\hline Triangle & $0.44 \leq$ & SF & $\leq 0.483$ \\
\hline Oval & $0.32 \leq$ & SF & $\leq 0.34$ \\
\hline Diamond & $0.36 \leq$ & SF & $\leq 0.38$ \\
\hline
\end{tabular}

Table 1: Represents some values of shape factor

We have tested our algorithm on some other photos as represented in Figure 4 and Figure 5 and our algorithm gave us perfect results.

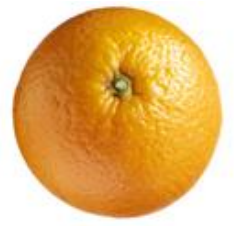

(A)

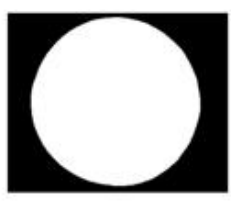

(B)

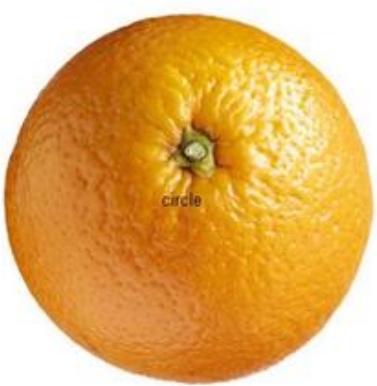

(C)

Fig. 4: A: Input Photo. B: Binary Photo. C: Detected and Recognized Shape 


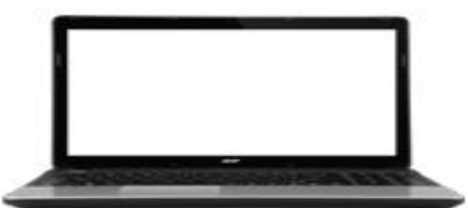

(A)

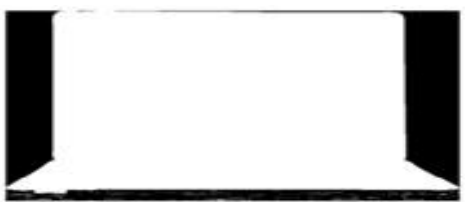

(B)

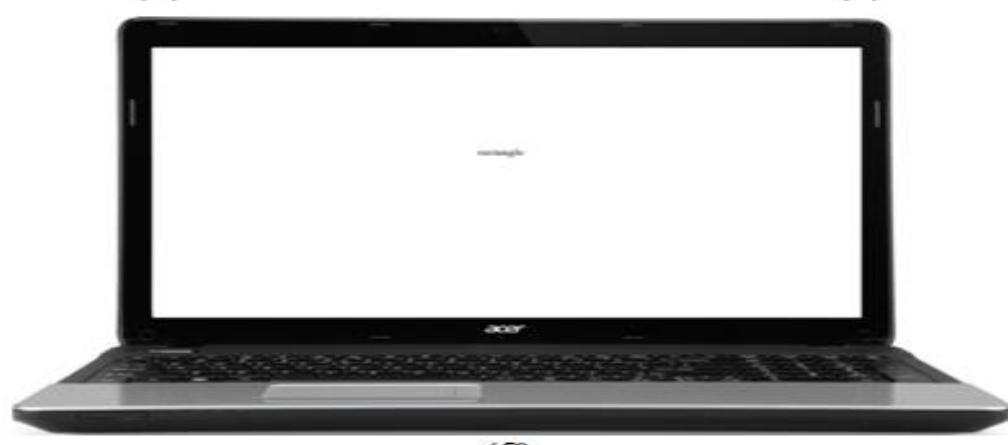

(C)

Fig. 5: A: Input Photo. B: Binary Photo. C: Detected and Recognized Shape

The process of our proposed algorithm in the current paper is just like follows:

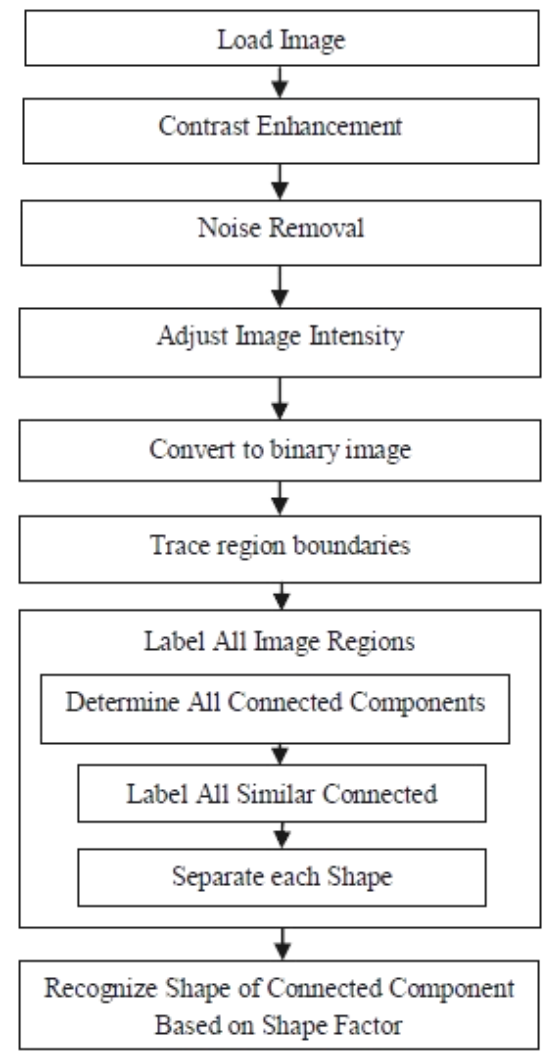

\section{Conclusions}

In the current paper we proposed a new algorithm to detect a shape from any photo and we could even recognize the kind of the shape given in the input photo and after applying our proposed algorithm on photos we saw that the algorithm gives very good results even if they are many shapes in one photo by depending on the value of the shape factor which is proposed in the current paper and if we compare our work with other works we could see that most of other works are focusing on detecting ang recognizing some specific shapes but our work is recognizing all the kinds of shapes and we can say that our system is working very well and gives good results finally. 


\section{References}

[1]. F. Pedro, "Representation and Detection of Shapes in Images," Massachusetts Institute of Tecnology, 2003

[2]. K. Karthik and ,. S. Wesley, "A Shape Recognition Algorithm Robust to Occlusion: Analysis and Performance Comparison," 2013.

[3]. K. M. S., "Shape recognition for image scene analysis," Université de Nice - Sophia-Antipolis, 2009.

[4]. M. Hankyu, C. Rama and R. Azriel, "Optimal Edge-Based Shape Detection," IEEE Transcations On Image Processsing NG, vol. 11, no. 11, pp. 1209-1226, 2002.

[5]. R. Mar, c. D. Philippe and L. Josep, "Boundary Shape Recognition Using Accumulated Length and Angle Information," Pattern Recognition and Image Analysis, vol. 4478, no. 27, p. 210-217, 2007.

[6]. S. Konrad and S. David, "Object Detection by Global Contour Shape," Article published in Pattern Recognition, pp. 1-30, 2008.

[7]. $\quad$ S. G., J. A., K. A., B. S., K. A, T. D.N. and N. A.K., "Object shape recognition from tactile images using regional descriptors," Nature and Biologically Inspired Computing (NaBIC), p. 53 - 58, 2012.

[8]. Z. M. Firdaus, C. H. Seng and S. S. Azmin, "Object Shape Recognition in Image for Machine Vision Application," International Journal of Computer Theory and Engineering, vol. 4, no. 1, pp. 76-80, 2012.

[9]. Q. Zhu, "SHAPE DETECTION BY PACKING CONTOURS," University of Pennsylvania, 2010 